slots unfilled and there was nothing in the information available at the point of allocation to differentiate service non-attenders from those who attended.

\section{Comment}

The limitations of this audit are acknowledged. The results are dependent upon the information provided by referrers and on clinicians completing audit forms in a consistent and comprehensive way. The problems referred may not necessarily be the focus of the work undertaken by CAMHS and often other problems emerge during the course of therapy. The audit has also undertaken a very pragmatic approach and has not attempted any social profiling nor focused upon matching indices of deprivation with referral patterns. Finally, in the absence of any comparable data it is not possible to determine whether these results are representative of other services.

Despite these limitations the audit has provided a wealth of information that has been helpful for clinicians, service managers and commissioning authorities. The results have been warmly welcomed by clinicians who feel that the complexity of their work is now better understood and their contribution valued. This has prompted a number openly to question and scrutinise their work resulting in the initiation of a number of separate audit projects. The service is more visible and more easily understood enabling service managers to examine how resources are currently allocated and to clarify purchaser expectations. The process has also facilitated change and has enabled teams to explore alternative ways in which they can respond to referrer demands. A summary of the audit has been sent to each commissioning authority providing them with a clear specification of the service they are currently commissioning. This in turn will provide an objective basis for future discussions about service priorities thereby enabling rational decision making about the use of existing resources. Finally, the audit has identified various shortfalls and the results may facilitate future developments and joint commissioning of services.

\section{References}

DEPARTMENT OF HEALTH AND DEPARTMENT FOR EDUCATION (1995) A Handbook on Child and Adolescent Mental Health. Manchester: HMSO.

KURTZ, Z (1996) Treating Children Well. A Guide to Using the Evidence Base in Commissioning and Managing Services for the Mental Health of Children and Young People. London: Mental Health Foundation.

- THORNES, R \& WOLKIND, S. (1994) Services for the mental health of children and young people in England. A national review. London: Department of Public Health, South Thames RHA.

NHS AdVISORY SERVICE (1995) Child and Adolescent Mental Health Services: Together We Stand. London: HMSO.

WALlACE, S. A., Crown, J. M.. Cox. A. D., et al (1995) Epidemiologically Based Needs Assessment: Child and Adolescent Mental Health. Winchester: Wessex Institute of Public Health.

*Paul Stallard, Clinical Psychologist and Robert Potter, Consultant Child and Adolescent Psychiatrist, Bath Mental Health Care Trust, Child and Family Therapy Service, Royal United Hospital, Combe Park, Bath BA1 3NG

*Correspondence

\title{
Accuracy of references in psychiatric literature: a survey of three journals
}

\section{A Lawson and Ruth Fosker}

\begin{abstract}
Aims and method The prevalence of errors in has not been reported as it has in other scientific reference citations and use in the psychiatric literature literature. Fifty references randomly selected from each
\end{abstract}


of three psychiatric journals were examined for accuracy and appropriateness of use by validating them against the original sources.

Results A high prevalence of errors was found, the most common being minor errors in the accuracy of citations. Major citation errors, delayed access to two original articles and three could not be traced. Eight of the references had major errors with the appropriateness of use of their quotations.

Clinical implications Errors in accuracy of references impair the processes of research and evidence-based medicine, quotation errors could mislead clinicians into making wrong treatment decisions.

A high prevalence of errors has been reported in the accuracy and appropriateness of references cited in some medical literature (de Lacey et al, 1985; Eichorn \& Yankauer, 1987; Hansen \& McIntire, 1994) but not yet in psychiatry. We present a survey that evaluated references in three psychiatric journals: the Psychiatric Bulletin (Bulletin), the British Journal of Psychiatry (BJP) and the American Journal of Psychiatry (AJP).

\section{The study}

All references cited in the February 1997 issues of the three journals were numbered consecutively and 50 chosen using a table of random numbers. These were checked for accuracy and appropriateness of their citations against the original sources obtained from the local postgraduate medical library or through normal inter-library loan in the UK. References not obtainable by these routes were classified as unverifiable.

Errors were classified as described by Eichorn \& Yankauer (1987) into major and minor. Major errors of citations were those that prevented immediate identification of the source of reference (e.g. errors in journal name, omission of year and volume and incorrect pages that did not overlap with correct pages). Minor errors in accuracy were misspellings, omissions or alterations of words in the title, omitted or incorrect sub-titles, incorrect page numbers that overlap with correct numbers, and omitted or incorrect authors' names, initials, or suffixes. Punctuation mistakes were not counted as errors.

Minor errors in the appropriateness of quotations included over-simplifications or generalisations not directly supported by the reference being cited. Major errors occurred when quotations were not substantiated by the original sources, contradicted them or were unrelated to them. An example of a major error occurred in a quotation that stiff baby syndrome is a severe neonatal form of hyperekplexia. This assertion was unsubstantiated by the original source which only suggested the possibility of a continuum between the two syndromes. In another example, a patient presented in hospital 45 minutes after an overdose of $1202 \mathrm{mg}$ risperidone tablets; the time to presentation was quoted as 45 hours which could mislead clinicians into believing that there was no urgency following the ingestion of a massive dose of risperidone.

When a reference was quoted more than once in an article, all assertions were checked for appropriateness and all errors recorded. However, when more than one quotation from the same reference was noted and the reference contained an error of citation, this was counted only once. All errors were otherwise counted and included in analysis. The relationship between the prevalence of errors and the number of cited references was studied using the quartile method (Eichorn \& Yankauer, 1987).

\section{Findings}

Forty-eight (96\%) of the 50 selected references were obtained from the AJP, 49 (98\%) from the Bulletin and all 50 references from the BJP $(100 \%)$. Errors in citation and quotations were found in all three journals. Table 1 shows the types and the prevalence of these errors in all the journals combined.

Table 1. Errors in accuracy and appropriateness of use in 147 references from three psychiatric journals

\begin{tabular}{lc}
\hline Type & $n(\%)$ \\
\hline Errors in accuracy of citations & \\
Minor & \\
Omitted/incorrect author initial or name & $30(20)$ \\
Omitted/incorrect word in title of paper & $6(4)$ \\
Omitted/incorrect subtitle of paper & $8(5)$ \\
Incorrect page numbers (overlapping) & $2(1)$ \\
Supplement not specified & $3(2)$ \\
Misspelt journal name & $2(1)$ \\
Major & \\
Omitted/incorrect journal volume or year & $2(1)$ \\
Incorrect page number (non-overlapping) & $3(2)$ \\
Errors in accuracy of quotations & \\
Minor & \\
Over simplification of reference & $0(0)$ \\
Generalisation from reference & $2(1)$ \\
Major & \\
Assertion not substantiated by reference & $4(3)$ \\
Assertion contradicted by reference & $2(1)$ \\
Assertion unrelated to reference & $2(1)$ \\
\hline
\end{tabular}




\section{Citation errors}

The AJP contained one (2\%) major error of citation and $17(35 \%)$ minor errors. The BJP had two (4\%) major errors and $22(44 \%)$ minor errors of citation while the Bulletin contained two $(4 \%)$ and $12(24 \%)$ respectively.

\section{Quotation errors}

Three references (6\%) in the AJP had quotations with major errors, no minor errors were found. Flve $(10 \%)$ references in the BJP had quotation errors classified as three $(6 \%)$ major and two $(4 \%)$ minor while the Bulletin had two $(4 \%)$ references with quotation errors, both major.

No relationship was found between the prevalence of citation or quotation errors and the number of references cited in the index articles of the journals individually or collectively.

\section{Comment}

The rate of minor errors in accuracy of citations is fairly high for the three journals studied and is comparable to prevalence rates previously reported for other types of journals (de Lacey et al, 1985; Eichorn \& Yankauer, 1987; Hansen \& McIntire, 1994). Omitted or incorrect authors initials or names remain the most common errors. These errors could result in an author not being properly acknowledged for his work.

Major citation errors delay or prevent location of the original reference making it difficult to verify the claim of the citing author(s) and impede further investigations by other readers (Goodrich \& Roland, 1977). The overall prevalence of major errors of citation in our study $(3 \%)$ is similar to those reported by Hansen \& McIntire (1994) in radiology journals and by Eichorn \& Yankauer (1987) in their study of three public health journals. The 1977 study by Goodrich \& Roland found a $20 \%$ error rate in the AJP compared with the $37 \%$ in our survey. Although the numbers of references verified in the two studies are different (i.e. 327 and 48, respectively), a real increase is suggested as similar criteria that can be objectively assessed were used in both studies.

Locating two of the cited references was delayed for several weeks due to major errors in their citations, however, the percentage of cited references that could not be verified was low (i.e.1\%), a figure smaller than in most other studies. In view of this, it would be inappropriate to expect journals to spend large resources on verifying the accuracy of reference citations. The responsibility for this rests with the author(s). but editors must ensure that authors carry out this obligation. Some journals require authors to provide documentation of all references before publication (i.e. part of, or the whole copy of a cited reference) (Hansel \& McIntire, 1994). In 1985 de Lacey et al suggested that journals should sample some references from each paper and to return a paper with any error back to the author with instructions to check all citations again.

Errors in appropriateness of quotations were less common and the combined rates for the three journals $(6 \%)$, were within the ranges previously reported for journals in other biomedical fields. That such errors have grave implications for research and evidence-based medicine is illustrated by Professor Davey Smith's recent letter to the British Medical Journal noting that he was twice misquoted in a study on serum cholesterol concentrations. depression and suicide (Davey Smith, 1997).

The use of multiple references in a sentence with multiple assertions has been found to be a source of quotation error (Eichorn \& Yankauer. 1987). We found only one instance of this practice which suggests it is diminishing.

The Bulletin has the lowest prevalence of citation and quotation errors. This may be due to a sampling error or the fact that articles in this journal were shorter and cited fewer references than did articles in the other two journals. However, the relationship between error rates and the number of cited references in an index article remains unclear; the existence of an association, and a lack of it has both been claimed by various studies. This study did not find an association.

Carelessness in reference citations could be indicative of a lack of discipline that also reflects the investigator's approach to other aspects of the research (i.e. research protocol, data management and laboratory practices in general) (de Lacey et al. 1985; Biebuyck, 1992). The practice of evidence-based medicine and research depends on ready access to appropriately quoted information. Errors in the accuracy and appropriateness of citations impair this process.

\section{References}

BIEвUYск. J. F. (1992) Concerning the ethics and accuracy of scientific citations. Anesthesiology. 77, 1-2.

DAVEY SMITH, G. (1997) Authors should read the literature that they cite (letter). British Medical Journal, 314, 144.

DE LACEY, G., RECORD. C. \& WADE. J. (1985) How accurate are quotations and references in medical journals? British Medical Joumal, 291. 884-886.

EICHORN. P. \& YANKAUER. A. (1987) Do authors check their references? A survey of accuracy of references in three public health joumals. American Joumal of Public Health, 77. 1011-1012.

GOODRICH. J. E. \& ROLAND. C. G. (1977) Accuracy of published medical reference citations. Joumal of Technical Writing and Communication, 7. 15-19. 
HANSEN, M. E. \& MCINTIRE D. D. (1994) Reference citations in radiology: accuracy and appropriateness of use in two major journals. American Journal of Roentgenology. 163, 719-723.

*L. A. Lawson, Senior Registrar in Forensic Psychiatry, Regional Secure Unit, Runwell
Hospital, The Chase, Wickford, Essex SS11 7QE; and Ruth Fosker, District Librarian, Colchester and North East Essex Postgraduate Medical Centre, Colchester General Hospital, Colchester.

\title{
The management of cylindrical battery ingestion in psychiatric settings
}

\author{
Nick Hindley, Harvey Gordon, Chris Newrith and Damian Mohan
}

\begin{abstract}
Aim and method To describe physical sequelae of cylindrical battery ingestion and their management, by description of recent cases and literature review. Results X-rays should be performed to determine the position and integrity of the battery. In the absence of abdominal symptoms, immediate surgical opinion may not be indicated.

Clinical implications Conservative management of battery swallowing is frequently possible.
\end{abstract}

There have been several recent episodes involving the swallowing of cylindrical batteries on the intensive care and admissions units at Broadmoor Hospital. People resident in these units have access to personal stereos which in almost all cases have been the source of the batteries which were subsequently swallowed. All episodes appear to have occurred at times of acute psychiatric disturbance and would seem to fit into the category of deliberate selfharm rather than frank suicidal intent. This particular behaviour appeared to follow a vogue over a period of two to three months before waning.

From the medical point of view, even psychiatrists well-acquainted with deliberate selfharm may be unfamiliar with the management of battery ingestion. Ingestion of foreign objects can create considerable anxieties and it can be that considerable pressure is put on the attending doctor to 'do something'. In such situations, prior knowledge of the potential sequelae of the act together with a clear picture of what reasonably needs to be done to ensure the person's safety can do much to diffuse such anxieties and to minimise the disruption caused both to and by the individual concerned.

The incidents encountered at Broadmoor provide some indication of the range of sequelae of battery swallowing and the extent to which management may vary according to the particular situation.

\section{Case illustrations}

\section{Patient 1}

A man in his twenties with a diagnosis of personality disorder and a long history of severe self-harm including self-laceration and persistent head-banging. During a period of acute disturbance he swallowed an alkaline cylindrical battery having bitten the casing to allow release of its contents. He became immediately symptomatic with acute upper gastrointestinal pain. Abdominal X-ray (AXR) showed a damaged battery in the stomach. He was treated as a surgical emergency and underwent laparotomy with removal of the battery. On return to Broadmoor he swallowed two further batteries in similar fashion resulting in a repeat laparotomy; 